\title{
RoonRoon: A Wearable Teddy as Social Interface for Contextual Notification
}

\author{
Fahim Kawsar $^{1}$, Kaori Fujinami ${ }^{2}$, Susanna Pirttikangas ${ }^{3}$, Keisuke Hayashi ${ }^{1}$, Tatsuo Nakajima ${ }^{1}$ \\ ${ }^{1}$ Department of Computer Science, Waseda University, Japan \\ ${ }^{2}$ Department of Computer Science, Tokyo University of Agriculture and Technology, Japan \\ ${ }^{3}$ Department of Electrical and Information Engineering, University of Oulu, Finland \\ \{fahim, tatsuo\}@dcl.info.waseda.ac.jp, fujinami@cc.tuat.ac.jp,msp@ee.oulu.fi
}

\begin{abstract}
In this paper we present a wearable teddy, RoonRoon ( $a$ physical embodiment artefact) that acts as a user interface for information services. It can monitor user's physical activity state (walking, standing, running and sitting) and can provide personalized information in a contextual manner minimizing interruptions. RoonRoon has four notification mechanisms: audible beeps, human voice, ambient sound and vibration. Since RoonRoon is intended primarily for mobile use, interaction is managed completely by voice using natural language. This paper describes the design and implementation of RoonRoon. We also present an informal study that reveals end users' impressions towards wearable toys as social interfaces.
\end{abstract}

\section{Introduction}

Moving off the paradigm of desktop computing seeks for new rational approaches for human computer interactions. Traditional user interfaces are not appropriate for the newly envisioned ubiquitous computing arena. Considering the collective and integrated participation of human and environment to provide personalized information pervasively, innovative, appealing and user-centric interfaces are very important. Smart phones and similar personal devices are getting major popularity for this purpose. However, beyond the primary role of a communication device, utilization of mobile phones is limited to messaging services (SMS, MMS, IM, etc.), lowend gaming and as a camera (a niche market). Albeit every other day new services are being integrated into mobile phones, the acceptance rate is very low [9]. One of the reasons is the stereotypical interfaces of mobile phone are just a miniature version of traditional computer without any additional appeal. More intuitive and aesthetic interfaces will perhaps attract more users to utilize the various information services that are coming up in mobile computing domain. In this paper, we investigate a wearable social interface through a context-aware embodiment teddy. Our primary motivation is to explore the possibility of adopting such context-aware wearable toys to information interface.

Based on our initial requirement study, we have selected two user-centric functions as the target service provisions: personal calendar and personalized news notification. As embodiment interface, we have selected a small teddy bear embedded with accelerometer sensor and vibrator. The teddy can monitor user's physical activity state (walking, standing, running and sitting) and can notify users about calendar events and subscribed news in a contextual manner using four different clues (audible beeps, human voice, ambient sound and vibration). It can analyze users' physical state to minimize the interruption of the notification. We name the teddy bear as RoonRoon and henceforth will use this term. We approach to model the behavior of this teddy bear into social interfaces using zoomorphic embodiments combined with anthropomorphic behavior. Considering, RoonRoon is primarily for mobile usage, users can configure, personalize and control this animatronics completely using speech.

The paper also presents the evaluation of RoonRoon by target user group that depicts some promising results. Generally, RoonRoon was appreciated although some users found speech (as communication medium) as annoying in public places. Also they preferred vibration to other notification mechanisms. Major implication of the evaluation is: People prefer to use wearable social interfaces if it is elegant and appealing regardless of the functional advantages.

\section{Motivation and Approach}

The motivation behind the creation of RoonRoon was boosted by the observation of minimal utilization of currently available multi-purpose mobile phones. Although attractive information services are being integrated into mobile phones, still it is used primarily as a communication platform. Anticipating this usage characteristic of current mobile phones, we have decided to explore the possibility of wearable toy as a sub-functional part of a mobile phone 
for information services. One observation that encourages us to pick teddy bear as candidate artefact is the fashion

trend of carrying different kind of teddy/ornaments as strap accessories for mobile phone in Japan and Korea as shown in figure 1. We have adopted this practice in our design and augmented a teddy bear that people can wear to delegate a few information services in a contextual manner.

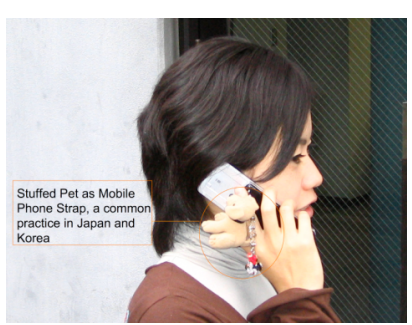

Figure 1. Teddy as Cell Phone Accessories
Our basic approach is to design these toys that interact socially and to model their interaction behavior at interface levels. In order for a teddy bear to be understood by humans it must have naturalistic embodiment and interact with its environment like regular toy [19]. We feel such interactions will be enjoyable for the users. Also because of its physical appearance and teddy like expressiveness it will have an appeal to people considering our natural liking tendency towards teddy bears. The animatronics is also "socially evocative" as it relies on our tendency to anthropomorphize and capitalize on feeling evoked when we nature, care or are involved with our creation and interaction [4], a primary reason of our liking towards teddy bears.

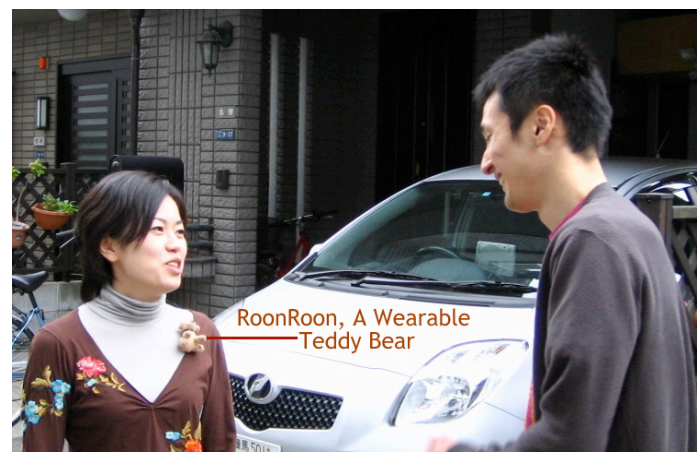

Figure 2. User Wearing RoonRoon

Taking these issues into account, we have built RoonRoon, embedded with accelerometer sensors and vibrators. Figure 2 shows a user wearing RoonRoon. It acts as a social interface for delegating the information by using various real pets like behavior like verbal speech, gesture (vibration) and verbal clue as music clip or audible clip. However, it has no social cognitive ness. It merely works according to the configuration that is being personalized by its owner.

\subsection{User Survey for Service Selection}

To identify the probable information services suitable for RoonRoon, we have conducted a user survey in a public sports center at Tokyo over a three days period. Total 103 people (55 Male, 48 Female, Age Range 10 60+) of 6 different categories based on their profession participated in the survey. We have presented them 12 hypothetical information services for next generation wearable devices and asked them for their preferred services in a 5-point scale. Figure 3 shows the participants' responses. Based on these responses we have selected two services for the initial version of RoonRoon. These are: Public Events (Personalized News, Ranked 1 with Mean: 4.35 and Standard Deviation: 0.39) and Personal Calendar (Schedule Notification, Ranked 2 with Mean: 3.87 and Standard Deviation: 0.11). Informal interview with a few participants revealed that the high score of Public Events/News are essentially because of the usefulness of timely delegation of specific information like earthquake, transportation problem etc. which are very common and important at Tokyo metropolitan area.

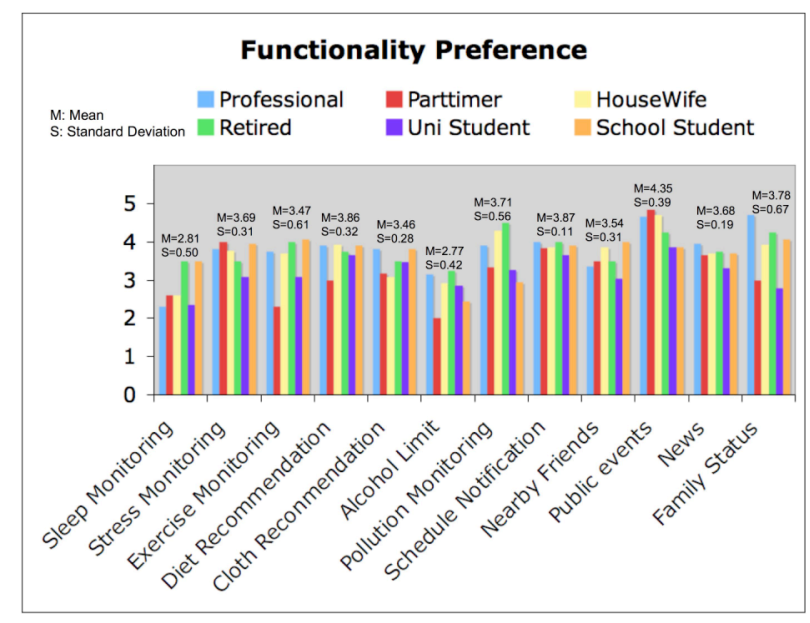

Figure 3: Survey Responses for Services

\section{Scenario and Functionality mapping}

In this section, we present a hypothetical scenario of the possible usage of RoonRoon followed by the functional mapping.

\subsection{Another Day for Elisa}

Recently one of Elisa's friends gave her a smart teddy named RoonRoon as a birthday gift. This morning when Elisa was going out for jogging, she clipped it on her Tshirt and activated it by saying. "Wake up". While she was running across the park RoonRoon told her "The weather will be quite warm this afternoon while appreciating her workout". Elisa returned home and while reading the newspaper, RoonRoon started beeping to notify her about an accident in the subway that she usually takes. After the breakfast she rushed out to school. As she approached the door RoonRoon started vibrating and reminded her that she forgot the birthday gift of Su. Elisa remembered that last night she updated her schedule and instructed RoonRoon for this event at this time. Later in the classroom while she 
was attending the art lecture, RoonRoon again activated and start plying an ambient sound to notify her about the latest movie listing. Elisa was disturbed and immediately deactivated it by saying: "Stop, I am busy now". After the lecture, she personalized RoonRoon not to activate in the classroom.

\subsection{Features and Functionality Mapping}

RoonRoon has several features that support the functionalities mentioned in the hypothetical scenario.

\section{RoonRoon has two major functions:}

1. It monitors users' physical activity and can identify different states (Running, Standing, Sitting and Walking) analyzing the data sensed by the accelerometer embedded into it.

2. It keeps track of users' schedule and can retrieve weather, earthquake, transportation and movie listing news from web. When an event occurs in user schedule or news are updated it notifies the user based on users preferred mode of notification and activity state to minimize interruption.

\section{RoonRoon has four notification mechanisms to notify any} events:

1. Audible Beep

2. Voice: It can talk in natural english language.

3. Ambient Sound: It can play a designated music clip.

4. Vibration: It can vibrate back part of its body.

\section{For Personalizing RoonRoon, users can:}

1. Designate mechanism to notify events, like whether to beep, talk, play music or vibrate to notify events.

2. Specify what RoonRoon should consider before activation. Like, if the user does not want to receive any information when he/she is walking/running/sitting/standing it can be configured accordingly.

3. Designate time period that RoonRoon should consider for its analysis of users activity.

4. Specify whether RoonRoon should be automatically activated when worn or manually activated when RoonRoon is being instructed by voice.

\section{For controlling RoonRoon, users can:}

1. Activate RoonRoon or can send RoonRoon to sleeping mode

2. Suspend RoonRoon's current action for later actuation

3. Immediately cancel RoonRoon's actions.

Table 1 summarizes the functional requirements for supporting these features. In the next section we discuss about the implementation of these functionalities.

\section{Implementation Detail}

Entire system has three major physical components:
1. A host machine that controls RoonRoon. Basically the host machine is connected to a sensor node and a headset using Bluetooth and can control the overall interaction (Remote Brain approach).

2. RoonRoon's physical body: A small teddy bear is augmented with a coin sized sensor node ${ }^{1}$ as shown in Figure 4. The sensor node contains a 2D accelerometer and a vibration motor and has Bluetooth connectivity.

3. A Bluetooth headset with microphone. We could attach the functionality within the RoonRoon body, but currently we have used a separate headset.

\begin{tabular}{|c|c|c|}
\hline \multirow{2}{*}{$\begin{array}{l}\mathrm{R} \\
\mathrm{O}\end{array}$} & Functionality & Mechanism Used \\
\hline & $\begin{array}{l}\text { Identifying } \\
\text { Physical State }\end{array}$ & $\begin{array}{l}\text { 2D Accelerometer sensor is } \\
\text { embedded in RoonRoon's body with } \\
\text { Bluetooth connectivity. }\end{array}$ \\
\hline $\mathrm{O}$ & $\begin{array}{l}\text { Tracking } \\
\text { Schedule }\end{array}$ & $\begin{array}{l}\text { iCalendar based scheduler and } \\
\text { scheduler parser. }\end{array}$ \\
\hline $\mathrm{N}$ & $\begin{array}{r}\text { Retrieving } \\
\text { Public Events }\end{array}$ & $\begin{array}{l}\text { Web services providing information } \\
\text { through RSS and RSS reader. }\end{array}$ \\
\hline $\mathrm{O}$ & $\begin{array}{c}\text { Voice } \\
\text { Interaction }\end{array}$ & $\begin{array}{l}\text { Speech Recognizer and Text to } \\
\text { Speech component. }\end{array}$ \\
\hline U & Music, Beep & Internal music player. \\
\hline 10 & Vibration & $\begin{array}{l}\text { Vibration motor is attached with } \\
\text { RoonRoon and is controlled remotely } \\
\text { through Bluetooth connectivity. }\end{array}$ \\
\hline
\end{tabular}

Table 1. Requirement Mapping to Functions

RoonRoon is built atop a generic middleware Prottoy $[7,8]$ for context aware computing. Prottoy has generic components that can encapsulate physical artefacts and/or context (sensor) information source and can present to the application in a unified way for manipulating the artefacts. Prottoy also has a Preference Manager component that provides unified interface for providing personalization and control facilities. This component has an integrated speech recognizer that has been used in RoonRoon for interacting with it and personalizing it. For further detail about Prottoy please consult the reference [7,8]. The architecture of RoonRoon is shown in Figure 5. All 6 functionalities, as mentioned in the table 1 are wrapped using Prottoy's component. The functional detail of the components are as follows:

1. 2D Accelerometer and Vibrator Wrapper: The coin sized sensor node containing 2D accelerometer is augmented with a vibrator. The accelerometer data is constantly being sent through Prottoy to the underlying host application where the data is analyzed accordingly using our custom algorithm [15]. When the vibration is needed, host machine generate specific command to this component through Prottoy.

\footnotetext{
${ }^{1}$ The sensor node is jointly developed by authors' laboratory and Nokia Research Center, Japan.
} 


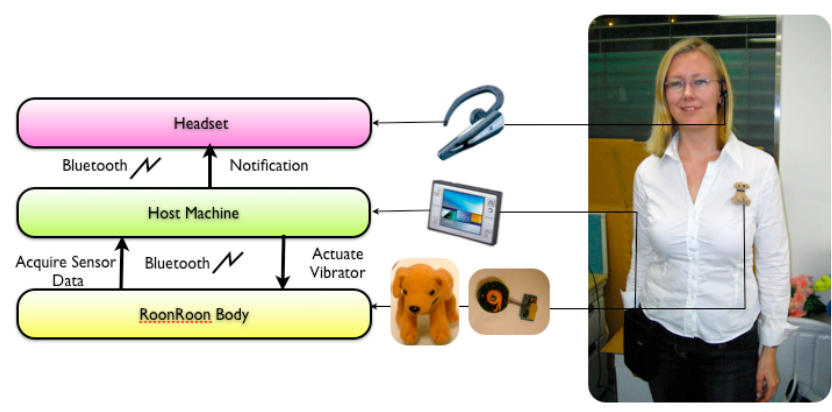

Figure 4. Physical Components of RoonRoon

2. Text to Speech Wrapper: This component is used to convert the schedule and news information into voice and also for using personalized statement used for notification.

3. Sound Wrapper: This component is used to play designated audio/music clip and beep to denote events.

4. News Wrapper: This component is used to retrieve public events from various websites using RSS. Whenever news is updated, user is notified taking users preference into account.

5. Schedule Wrapper: This component is used to extract the schedule information from an iCalendar-based scheduler. The scheduler is running in the host machine.

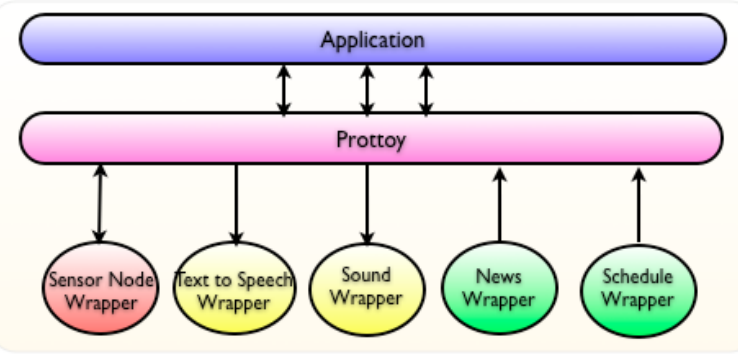

Figure 5. Basic Architecture of RoonRoon

All these components run in the host machine. Host machine interacts with RoonRoon and headset using Bluetooth for extracting sensor information and relaying notification.

\subsection{States of RoonRoon}

Basically RoonRoon has two states: Active Mode and Sleeping Mode. In active states, it starts monitoring the movement pattern and polling schedule and news. RoonRoon can be set to this mode manually by voice command or automatically after startup. Users can send RoonRoon to sleeping mode state by voice command. In this mode RoonRoon does neither monitor activities nor track schedule information.

\subsection{Interaction with RoonRoon}

For interacting with RoonRoon user need to use the predefined phrases that RoonRoon understands. The speech recognizer identifies the phrases and presents it to the application running on the host machine. Users can completely configure and personalize RoonRoon as mentioned in functionality mapping section using predefined phrases. Based on the analysis of perceived information (accelerometer sensor data and schedule information) the application decides what actions to perform and accordingly uses text to speech component for uttering statement or music player component to play music or beep (both through the headset) or sensor component to vibrate the body of RoonRoon.

\subsection{Technical Detail}

RoonRoon is entirely written in Java on top of Prottoy which is also Java based. For text to speech we have used a Java based library called FreeTTS [5]. For Bluetooth communication we have used Java JSR82 APIs implemented in Aventa [1] library. In Prottoy, for speech recognition Sphinx [13] has been used.

\section{Evaluation}

To evaluate the performance and acceptability of RoonRoon we have conducted a user study participated by 15 people from 7 different countries. In this section we present our study results.

\subsection{Experiment Procedure}

Initially RoonRoon was introduced through a formal presentation. A command manual to interact with RoonRoon was given to them and was requested to go through. Then the evaluator demonstrated example usage by interacting with RoonRoon. Finally participants were invited to use. Each trial took about 30 40 minutes. After that, participants were presented a questionnaires followed by an interview. The participants' profiles are mentioned in table 2 .

\begin{tabular}{|c|c|l|}
\hline Participants & Age & \multicolumn{1}{|c|}{ Profession } \\
\hline 9 Female & $23 \sim 35$ & $\begin{array}{l}\text { Graduate Student, Lawyer, } \\
\text { Social Researcher, Business } \\
\text { Consultant. }\end{array}$ \\
\hline 6 Male & $25 \sim 52$ & $\begin{array}{l}\text { Graduate Student, Professor, } \\
\text { Engineer, Researcher. }\end{array}$ \\
\hline
\end{tabular}

Table 2. Demography of the Participants

\subsection{Guiding Topic}

We have designed the questionnaires and the interview following the guideline topics mentioned below:

1. Functional Features: Whether the participants like the functions of RoonRoon? 
2. Notification Mechanism: How appropriate the actuation mechanisms are?

3. Interaction and Personalization: Whether the participants are satisfied with the Interaction and personalization features?

4. Masculine/Feminine Feature: Whether RoonRoon appearance is gender free?

5. Unobtrusiveness

6. Usefulness

7. Usability

\subsection{Quantitative Results}

Since some the questions were very direct in the form of affirmative and negative response, we have requested the participants to rate their answers on a 5-point scaling. In the following figure 6-7 results are shown.

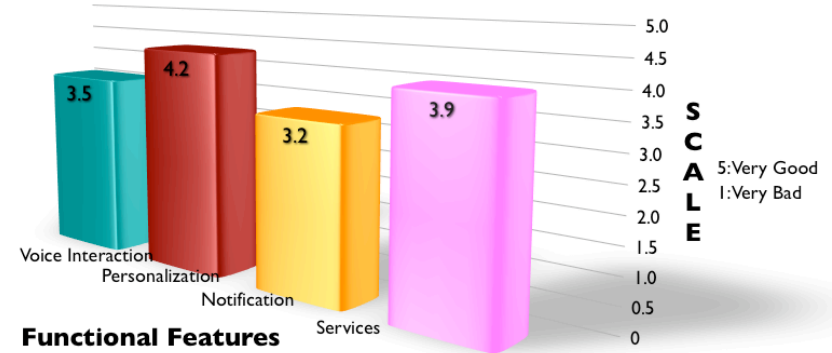

Figure 6. Users' Responses on Funcational Features

As shown in Figure 6, personalization feature of RoonRoon was appreciated, similarly as were the services that we have picked from our initial pilot study. There were mixed reactions on voice-based interaction, specifically some users told us that it is completely unrealistic to talk to a teddy in public places. However they agreed that for wearable applications voice commands provide better usability. From notification perspectives, the average scaling was not very promising. The negative biasing factor were the speech and sound notifications as most of the users found it obtrusive and inappropriate. The response graph is shown in Figure 7. Vibration was preferred as the most appropriate and less obtrusive notification scheme followed by audible beep.

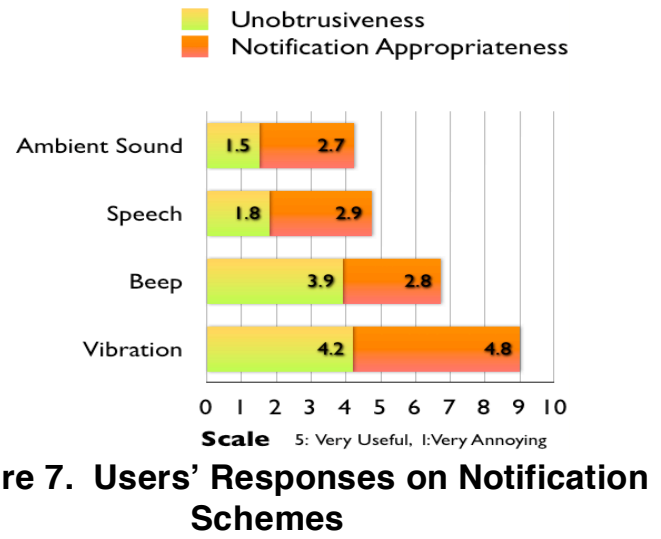

\subsection{Qualitative Results}

In general, all the participants grasped the notion of RoonRoon and its functionalities. Figure 8 shows the overall user response on RoonRoon's quality aspects. Overall usability and unobtrusiveness of RoonRoon is quite promising. However, some of the users were not very convinced by the overall usefulness of RoonRoon. As they feel, more services should be incorporated to make it attractive as a product. Mere news and schedule notification

is not enough to motivate them to carry a teddy all the time. A specific comment from the informal interview that summarizes this view was

"I don't still realize what special advantage I will have. I don't want to wear it when I am teaching in the class for example, just for schedule and news."

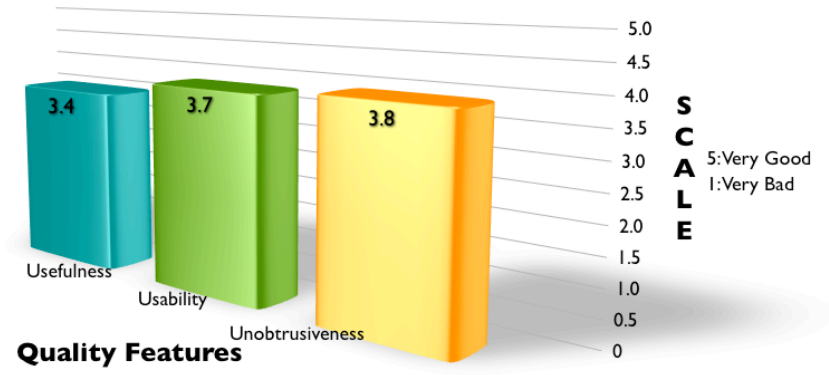

Figure 8. Users Responses on Quality Features

Another aspect that was revealed during the user study that current RoonRoon appearance is definitely feminine.

Users response as shown in figure 9 confirms this fact where $71 \%$ users think it's a feminine accessories. Several male participants commented that they prefer to have this services in more generic accessories, one comment was "At least this teddy is not for us,

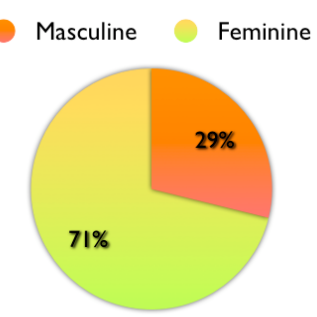

Appearance Attribute

Figure 9. Appearance Responses may be a pen, a watch or similar things are ok for me". Interestingly female participants felt the cute appearance was the motivation factor considering it might be used as a broach if it matches their overall style. One comment was "May be making it more fashionable might motivate us to clip it on to our clothes. Otherwise I usually would not want to clip a teddy on my clothes unless it matches my overall style".

\subsection{Summary of the Evaluation}

In general, participants liked cute appearance of RoonRoon, however were not very convinced speech based interaction feature, as it appeared to be obtrusive and 
inappropriate. Users prefer vibration to other notification schemes. The dynamic personalization facilities of RoonRoon were well appreciated. One interesting finding is that none of the participants pointed about the integration of these functionalities into a mobile phone. Intentionally we keep this topic out of the questionnaires. However when being asked about enhanced mobile phone with such features, we have received some interesting comments. Two of them are mentioned below.

"Well, it would be nice to have this feature. But I am wondering what is the goal of this toy, if it is just a smart toy then it should be like this."

"To me, this toy has a different appeal that is missing in cell phone, although we may have some of these functions in the phone, we rarely use them. This kind of toy might be compelling for using those functions like RoonRoon has"

Considering this viewpoint, we believe the choices to be made here is between the aesthetics that the physical embodiment artefact provides and the enhanced cell phone features. Taking our initial observations of mobile phone accessories into account, we argue that elegant, cute and emotionally evoked interfaces are often more satisfactory and important than stereotypical interfaces with enhanced functionalities. However considering user responses in this study, it can be implied that such wearable teddy may not be welcomed only for functional features but for elegant and cute appearances. Our claim is also justified by $[10,11]$.

\section{Discussion and Future Work}

From system performance point of view RoonRoon is found to be stable. RoonRoon provides user with the complete personalization and control facilities. Users can configure the functionalities and notification mechanism according to their own preferences. Users preferences and context awareness together minimizes the interruption and instigate timely actuation. However, we recognize proposing speech-based interactions with stuffed animatronics are non-convincing because it is not natural. The users' evaluation confirms this fact. We believe this finding is very crucial for such wearable animatronics as the alternatives are very few and limited to gesture or tagbased interaction. Embedding typical GUI in these animatronics is not natural and will violate the natural appearance. It would be interesting to see how users react to these interaction techniques for social interfaces like RoonRoon.

Context-aware behaviour makes RoonRoon more socially evocative. Several participants in our study commented that RoonRoon's behaviour is appropriate in a quiet place. One enhancement could be improving the unobtrusiveness quality by incorporating social proximities i.e. presence of someone with the wearer. We are working on adding this feature to increase RoonRoon's use in public spaces. Another enhancement that we are currently working on is to add location awareness to the personal calendar so that calendar events can be notified at appropriate locations. RoonRoon's functions are not tightly coupled with the teddy bear appearance. Since the architecture of RoonRoon is modularized, we can easily accommodate any other similar wearable artefact whose metaphor is diverse enough to express the functional features. Our informal study revealed that the current appearance of RoonRoon is feminine. However, a pen, a watch or similar unisex accessories can be augmented to make RoonRoon's appearance gender-free.

The selection of the metaphor is very crucial for social interfaces. For example: talking to a pen/watch is completely unnatural. Some of the evaluators mentioned that talking to a teddy is also unnatural. However, we argue that the degree varies depending on the physical appearances and our natural understanding. Most of the lively pet owners talk to their pet in natural English though the feedback is never in human linguistics (except some talkative birds, like a parrot etc.). The point we trying to make here is, it is very important to select a metaphor that conforms to our natural understanding. This is an interesting topic for the social interface designers. Several research groups are working on this issue so we are hoping that soon we will have a have a guideline to select appropriate metaphor for social interfaces.

\section{Related Work}

Physical embodiment interfaces have been investigated in various researches. One of the early works in this area is the COMRIS (Co-Habited Mixed Reality Information Spaces) parrot by Van de Velde [16] and De Haan [3] where the parrot acts as a wearable advisor. Its focus domain is large public gathering like conference, exhibition etc. It can suggest the wearer about interesting events. Van de Velde also investigated the applicability and effectiveness of such wearable physical artefact advisors. RoonRoon is different from the point of view that it does not attempt to suggest user with any moments of interest rather it functions to notify user about events in a context aware manner analyzing users activities.

A closely related domain is the Robotic User Interface, where the stereotypical robots are used to integrate the physical and digital world [2]. Greenberg et al. suggested the digital representation of people as avatars in the office environment [6]. Our work is entirely distinctive from this work considering we focus on physical social interface with effective functional behavior. Several electronics companies have developed various pet robots like Sony AIBO [17], Matsushita Tama etc. with verbal and gesture facilities for entertainment. However our work is different from these initiatives considering the fact that rather than providing interface with cognitive capability for entertainment, we focus on delegating sensor perceived useful information to social interfaces built using physical tangible artefact with appropriate human understandable verbal/non verbal clues. Also our major concern is to give an elegant look to the physical embodiment rather than the metallic view of robots. Similar to our work is the sensor doll built by Yonezawa et al. [18]. The doll is augmented 
with multimodal interface and can react to external stimuli by playing music. Although the work is very similar to ours, RoonRoon is more user-centric. Rather than a general toy, RoonRoon has a purpose of analyzing users' states to provide useful information. Additionally RoonRoon can interact with voice and can make gestures through vibration. Two other physical embodiment works are Robot-PHONE [12] and Cellular Squirrel [14]. Both works use stuffed animal like squirrel, bunny, teddy bear etc. as a physical interface that attempts to replicate the functionalities of a mobile phone. Our work is different from the functional point of view, as we are not focusing on synchronizing with the cellular phone. RoonRoon's distinctive attributes from others' work are its context aware functional features, unique personalization and controlling facilities with a cute and aesthetic appearance.

\section{Conclusion}

RoonRoon is designed as a social interface for delegating pervasive information services. Our hypothesis was that such social interfaces would attract more people to use context aware services in their daily lives since they promises to be more socially evocative. However, our study reveals that aesthetics and emotional appeals play the important role for the success of such social interfaces rather than the funcational features. People consider these wearable toys as ornaments or fashion accessories. The computation functions are just value additions. Perhaps the important challenge is to identify how to integrate the value added services into these teddy bears or other social interfaces in a way that the physical appearances and functional features become inseparable. We are focusing on this usability issue and hope soon we will come up with some interesting results.

\section{References}

[1] Aventa URL: http://www.aventa-gmbh.de

[2] Bartneck, C., Okada, M. Robotic User Inter-faces. The Human and Computer Conference (HC-2001), Aizu, 2001

[3] De Haan, G. The Usability of Interacting with the Virtual and the Real in COMRIS. In TWLT 15 - Interactions in Virtual Worlds, 1999
[4] Fong, T., Nourbakhsh, I., and Dautenhahn, K. A Survey of Socially Interactive Robots: Concepts, De-sign, and Applications. Technical report CMU-RI-TR-02-29, Robotics Institute, CMU, 2002

[5] FreeTTS URL: http://www.freetts.sourceforge.net/

[6] Greenberg, S. and Kuzuoka, H. Using Digital but Physical Surrogates to Mediate Awareness, Communication and Privacy in Media Spaces. Personal Technologies, 4(1), 2000

[7] Kawsar. F., Fujinami. K, and Nakajima. T., "Design and Implementation of a Software Infrastructure for Integrating Sentient Artefacts", The 2nd International Conference on Mobile and Ubiquitous Systems: Networking and Services (Mobiquitous2005), San Diego, USA, July 2005.

[8] Kawsar. F., Fujinami. K, and Nakajima. T., "A Middleware for Sentient Environments", The 2005 IFIP International Conference on Embedded And Ubiquitous Computing (EUC-05), Nagasaki, Japan, December 2005.

[9] Lemelson-MIT Invention Index study, 8th annual, January 21, 2004,

[10] Norman, D.A., "The Design of Everyday Things". NY: Basic Books, New York, 2002.

[11] Norman, D.A., "Emotional Design”. NY: Basic Books, New York, 2004.

[12] Sekiguchi, D., Inami, M., Tachi, S. Robot-PHONE: RUI for Interpersonal Communication. Ex-tended Abstracts of CHI 2001, 2001

[13[ Sphinx URL: http://www.cmusphinx.sourceforge.net/

[14] Stefan, M., Schmandt, C., Physical embodiments for mobile communication agents, 18th Annual ACM Symposium on User Interface Software and Technology, Seattle, 2005

[15] Pirttikangas, S., Fujinami, K, Nakajima, T., Feature Selection and Activity Recognition from Wearable Sensors, 2006 International Symposium on Ubiquitous Computing Systems (UCS 2006), Korea 2006.

[16] Van de Velde, W. Co-Habited Mixed Reality. Fifteenth International Joint Conference on Artificial Intelligence (IJCAI97), Japan. 1997

[17] Website of Sony AIBO: http://www.eu.aibo.com/

[18] Yonezawa, T., Clarkson, B., Yasumura, M., Mase,K. Context-aware Sensor-Doll as a Music Expression Device. Extended Abstracts CHI'01, 2001

[19] Zlatev, J. The Epigenesis of Meaning in Human Beings and Possibly in Robots. Minds and Machines 11(2), 2001 